\title{
Cost-effective HIT diagnosis: utilizing IgG- specific PF4 immunoassays reduces the number of confirmatory serotonin release assays without missing true HIT
}

\begin{abstract}
In the United States, the most commonly employed screening assay for heparininduced thrombocytopenia (HIT) is the PF4 ELISA, of which both polyclonal (poly-ELISA) and IgG-specific (IgG-ELISA) assays are commercially available. We compared the IgG-ELISA versus the poly-ELISA to determine whether the IgGELISA is more sensitive and specific for the diagnosis of HIT. 453 HIT work-ups were reviewed. Patients with poly-ELISA optical density (OD) values greater than or equal to $0.40(n=49)$ were selected for further analysis, including serotonin-release assays (SRAs), IgG-ELISAs, and pre-test probability scoring (4T's score). Both the poly-ELISA and IgG-ELISA identified PF4/heparin antibodies in all true HIT patients $(\mathrm{n}=8)$. IgG-ELISA reduced the number of "borderline" cases by $75 \%$. IgG-ELISA is more specific than poly-ELISA and can reduce number of confirmatory SRA required.
\end{abstract}

Volume I Issue I - 2015

\author{
Oksana Volod, Sumire Kitahara, Navid \\ Farahani \\ Department of Pathology and Laboratory Medicine, Cedars \\ Sinai Medical Center, United States
}

Correspondence: Oksana Volod, Department of Pathology
and laboratory Medicine, Cedars Sinai Medical Center ,8700
Beverly blvd. Room 47II, Los Angeles U.S.A, Tel ( 310$) 423$ Beverly blvd. Room 47II, Los Angeles U.S.A, Tel ( 310$) 423-$
547I, Fax ( 310 ) 423 - 0483, Email Oksana.Volod@cshs.org

Received: April 22, 2015 | Published: May 28, 2015

Keywords: IgG-ELISA, false positive, CAP-PT, IgA, hematopathology

Abbreviations: HIT, heparin-induced thrombocytopenia; PF4 ELISA, platelet factor4 enzyme-linked immunosorbent assay; SRA, serotonin release assay; OD, optical density; CAP-PT, college of American pathology proficiency testing; DTI, direct thrombin inhibitors; Poly-ELISA, polyclonal immunoassay; ISTH, international society on thrombosis and hemostasis; ICU, intensive care unit; TAT, turnaround time

\section{Introduction}

Roughly 12 million patients-or one third of hospitalized patients have some heparin exposure annually. ${ }^{1}$ Although a subset of patients will develop anti-platelet factor 4 (PF4) complex antibodies, only up to $10 \%$ of this subset ${ }^{2,3}$ (approximately 1-3\% of all patients receiving the drug for at least 7days) will develop clinically relevant, plateletactivating antibodies leading to the development of heparin-induced thrombocytopenia (HIT), with or without thrombosis, further referred to here as true HIT. ${ }^{1}$ In our previous study, we showed that the overdiagnosis of HIT is particularly endemic in medical and cardiac intensive care units, resulting in the over-utilization of direct thrombin inhibitors (DTIs), as an alternative method of anticoagulation, by a rate that exceeds the true incidence of HIT by an order of magnitude or more. ${ }^{4}$

Because HIT is a clinicopathological diagnosis, laboratory testing plays a crucial role in its definitive diagnosis. Currently, most laboratories utilize an immunoassay (or antigen test) as a screening modality. According to a recent College of American Pathology Proficiency Testing (CAP-PT) survey, there are two major types of anti-PF4/heparin ELISAs that are employed by many U.S. laboratories: a polyclonal immunoassay (poly-ELISA) that detects antibodies of all classes (IgA, $\operatorname{IgM}$, and $\operatorname{IgG}$ ) and an $\operatorname{IgG}$-specific immunoassay (IgG-ELISA). The poly-ELISA has historically been the screening test of choice for many laboratories, however, there is a growing body of evidence that IgG antibodies are the dominant, and perhaps the only clinically relevant immunoglobulin capable of inducing the platelet activation responsible for HIT. ${ }^{5-7}$ Many experts believe that a confirmatory test isn't necessary if there is a high clinical suspicion and the screening immunoassays (ELISAs) are positive in relatively high titers. However, in our experience, we have seen many confounding variables, especially in ICU and cardiac patients; ${ }^{8}$ such that we believe confirmation testing is necessary to establish the specific platelet activating properties of antibodies. This approach will be more cost effective and safer than using heparin alternatives in the management of patients who only have positive screening assay. In the context of our large quaternary care center, we sought to determine if the IgG-ELISA could serve as an improved screening assay over the poly-ELISA to reduce the rate of false positives, classically associated with any screening assay.

\section{Materials and methods}

\section{Patient population}

The following study was approved by the institutional review board of Cedars-Sinai Medical Center. Sera from 453 consecutive surgical/ medical patients who received unfractionated or low-molecularweight heparin, and in whom HIT was suspected or to be ruled out, were evaluated in this study. The patient samples were referred for diagnostic HIT testing to our laboratory between March 2011 and February 2012. The combination of a negative poly-ELISA with a low pre-test probability has a high negative predictive value for HIT, and therefore, these cases were excluded. Thus, of the original 453 samples, 86 samples with poly-ELISA OD's $\geq 0.40$, from 49 different patients (multiple work-ups performed on some patients) exposed to unfractionated heparin were chosen for additional testing for the purposes of this study. When multiple work-ups were submitted on the same patient, the sample with the highest result was selected for evaluation in this study. In-house IgG-ELISA and confirmatory sendout SRA were performed on these samples. Additionally, all workups 
included pre-test scoring using the Warkentin et al. ${ }^{2}$ 4T's scoring model.

\section{Assessing the pre-test probability}

In order to assess the pre-test probability of HIT, pathology residents rotating on the coagulation service evaluated patients by utilization of the Warkentin et al. ${ }^{2}$ 4T's clinical scoring model, including: thrombocytopenia, timing of platelet fall, presence of thrombosis, and alternative possible causes of thrombocytopenia. The patients were scored and their respective total scores calculated based on this system, as follows: 0-3: Low probability of HIT, 4-5: Intermediate probability of HIT, 6-8: High probability of HIT. The scores were then verified by one of two hematopathology attending physicians with extensive experience in the field of coagulation.

\section{PF4 immunoassays}

Both PF4 immunoassays were performed at Cedars-Sinai Medical Center. These commercially available tests (GTI Diagnostics, San Diego, CA) include:

i. A polyclonal anti-PF4/heparin ELISA that detects antibodies of three immunoglobulin classes $(\operatorname{IgG} / \operatorname{Ig} \mathrm{A} / \operatorname{IgM})$ against PF4/ polyvinyl sulfonate complexes

ii. An IgG-specific anti-PF4/heparin ELISA that detects antibodies of the IgG immunoglobulin class only.

For the poly-ELISA, the manufacturer recommends reporting a dichotomous result as positive or negative based on an optical density (OD) of greater than or equal to 0.40 . Based on a prior inhouse validation study (presented at the ISTH Congress in 2007), our laboratory additionally reports the following interpretative numerical OD values for the poly-ELISA: "negative" $=0-0.39$; "borderline" $=0.4-0.84 ; \quad$ "positive" $=0.85-2.79 ; \quad$ "strongly positive" $\geq 2.80$. The additional categories were developed as a result of the aforementioned validation study (unpublished results), which revealed that only $\sim 10 \%$ of patients within the "borderline" category, $\sim 20 \%$ of patients with the "positive" category, and $~ 50 \%$ of patients within the "strongly positive" category develop true HIT. For the IgGELISA, the manufacturer recommends reporting a dichotomous result as positive or negative based on an $\mathrm{OD} \geq 0.40$.

\section{Serotonin release assays}

The serotonin release assay is a complex, radio labeled test performed at a select number of specialized laboratories; our samples were sent to the Blood Center of Wisconsin (BCW). SRA results are reported by the $\mathrm{BCW}$ as follows: negative if both low-dose $(1 \mathrm{U} / \mathrm{mL})$ and high-dose $(100 \mathrm{U} / \mathrm{mL})$ concentrations of heparin show $<20 \%$ of serotonin release, positive if low-dose heparin concentration shows $>20 \%$ serotonin release \& high-dose heparin concentration shows $<20 \%$ serotonin release. The percent release with low and high dose heparin are reported by the Blood Center of Wisconsin; the results were then interpreted by two hematopathology attending physicians with extensive experience in the field of coagulation and labeled as either "positive" or "negative".

\section{Results}

\section{Comparison of poly-ELISA versus IgG-ELISA in relation to SRA}

Table 1 shows the results from each of the 49patients who were included in this study, including poly-ELISA and IgG-ELISA results, and SRA results. A high pre-test probability of HIT was present in
$7(14 \%)$ patients, an intermediate pre-test probability was present in $19(39 \%)$ patients, and a low pre-test probability was present in $23(47 \%)$ patients. Figure 1demonstrates that out of the 49patient samples, 29patients had "borderline" poly-ELISA ODs (0.4-0.84); the corresponding range of IgG-ELISA ODs was 0.07-0.73 and none of the cases were SRA-positive; 19 patients had "positive" poly-ELISA ODs (0.85-2.79); the corresponding range of IgG-ELISA ODs was 0.29 to $>3.0$ and 7 of the 19 cases were SRA-positive; 1patient had a "strong positive" poly-ELISA OD ( $\geq 2.80)$; the corresponding IgGELISA OD was 1.86 and the case was SRA-positive. Among 40 patients with negative poly-ELISA and IgG-ELISA (data not shown), the mean IgG-ELISA results was $0.6 \mathrm{OD}$ with a variance and standard deviation of 0.4 and $0.6 \mathrm{OD}$, respectively.

Table I A comparison of poly-ELISA versus IgG-ELISA in relation to SRA

\begin{tabular}{|c|c|c|c|c|}
\hline Patient & $\begin{array}{l}\text { PF4 } \\
\text { [polyclonal] }\end{array}$ & $\begin{array}{l}\text { PF4 } \\
\text { [lgG] }\end{array}$ & $\begin{array}{l}\text { SRA } \% \\
\text { low dose }\end{array}$ & $\begin{array}{l}\text { SRA } \% \\
\text { high dose }\end{array}$ \\
\hline 1 & 3.12 & 1.86 & 98 & I \\
\hline 2 & 2.58 & 2.02 & 43 & 0 \\
\hline 3 & 2.5 & 2.6 & 89 & 0 \\
\hline 4 & 2.47 & 3.26 & 100 & 2 \\
\hline 5 & 2.42 & 2.42 & 1 & I \\
\hline 6 & 2.25 & 1.89 & 44 & 0 \\
\hline 7 & 2.15 & 2.47 & 0 & I \\
\hline 8 & 2.09 & 2.14 & 5 & 4 \\
\hline 9 & 1.89 & 2.94 & 50 & 0 \\
\hline 10 & 1.86 & 0.53 & 2 & 0 \\
\hline 11 & 1.8 & 2.39 & 2 & 3 \\
\hline 12 & 1.62 & 0.96 & 0 & 0 \\
\hline 13 & $1.4 \mid$ & 1.92 & 86 & I \\
\hline 14 & 1.25 & 1.79 & 0 & 0 \\
\hline 15 & 1.23 & 0.87 & 2 & 0 \\
\hline 16 & 1.18 & 1.62 & 91 & 0 \\
\hline 17 & 1.16 & 1.00 & 0 & 11 \\
\hline 18 & 1.11 & 1.5 & 0 & 0 \\
\hline 19 & 1.08 & 0.7 & 1 & 0 \\
\hline 20 & 0.92 & 0.29 & 1 & 3 \\
\hline 21 & 0.83 & 0.43 & 1 & 0 \\
\hline 22 & 0.81 & 0.73 & 1 & 0 \\
\hline 23 & 0.79 & 0.11 & 0 & I \\
\hline 24 & 0.77 & 0.36 & 1 & 0 \\
\hline 25 & 0.72 & 0.63 & 5 & I \\
\hline 26 & 0.71 & 0.39 & 24 & 12 \\
\hline 27 & 0.57 & 0.43 & 0 & 0 \\
\hline 28 & 0.56 & 0.12 & 0 & 0 \\
\hline 29 & 0.56 & 0.11 & 2 & 1 \\
\hline 30 & 0.56 & 0.07 & 0 & 0 \\
\hline
\end{tabular}


Table Continued....

\begin{tabular}{|c|c|c|c|c|}
\hline Patient & $\begin{array}{l}\text { PF4 } \\
\text { [polyclonal] }\end{array}$ & $\begin{array}{l}\text { PF4 } \\
{[\operatorname{lgG}]}\end{array}$ & $\begin{array}{l}\text { SRA \% } \\
\text { low dose }\end{array}$ & $\begin{array}{l}\text { SRA \% } \\
\text { high dose }\end{array}$ \\
\hline 31 & 0.55 & 0.51 & 0 & 0 \\
\hline 32 & 0.53 & 0.3 & 83 & 63 \\
\hline 33 & 0.51 & 0.35 & 14 & 12 \\
\hline 34 & 0.49 & 0.15 & 0 & 5 \\
\hline 35 & 0.48 & 0.64 & 1 & 0 \\
\hline 36 & 0.47 & 0.14 & 4 & 5 \\
\hline 37 & 0.47 & 0.12 & II & 11 \\
\hline 38 & 0.46 & 0.21 & I & 0 \\
\hline 39 & 0.46 & 0.23 & 0 & 0 \\
\hline 40 & 0.45 & 0.11 & I & 4 \\
\hline 41 & 0.43 & 0.28 & 0 & 0 \\
\hline 42 & 0.43 & 0.13 & 0 & 0 \\
\hline 43 & 0.43 & 0.1 & 0 & 0 \\
\hline 44 & 0.43 & 0.17 & 0 & I \\
\hline 45 & 0.42 & $0.4 I$ & I & 0 \\
\hline 46 & 0.41 & 0.26 & 0 & 0 \\
\hline 47 & 0.41 & 0.18 & 2 & 0 \\
\hline 48 & $0.4 I$ & 0.17 & 0 & 0 \\
\hline 49 & 0.4 & 0.21 & I & 2 \\
\hline
\end{tabular}

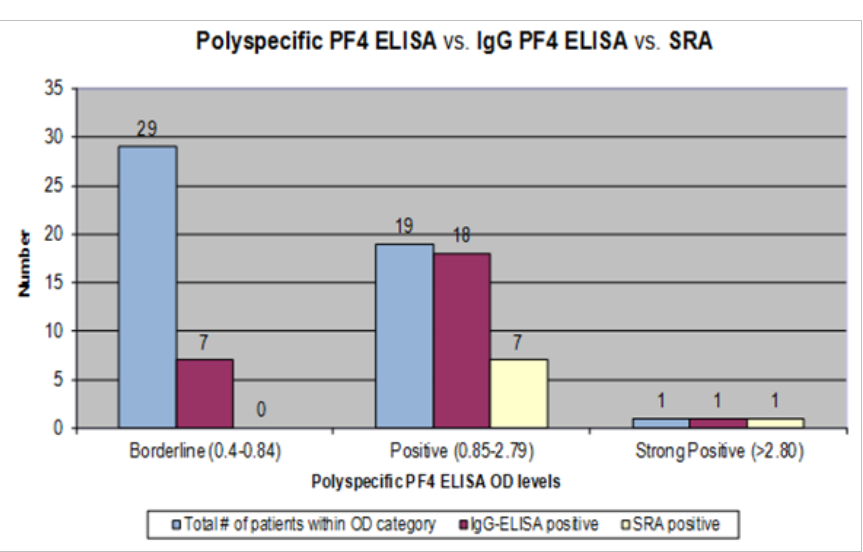

Figure I Interpretation categories by polyspecific ELISA, IgG-ELISA and SRA. Blue bars indicate the total number of patients within each category (borderline, positive or strong positive) by the poly-ELISA. Magenta bars indicate the total number of patients that resulted in the designated OD values by IgG-ELISA. Yellow bars indicate patients with positive SRA within each category.

\section{Sensitivity, specificity \& predictive capabilities of immunoassays}

True positives (or true HIT) were defined as patients with plateletactivating antibodies demonstrated by both immunoassays (i.e. PF4 ELISA) and confirmatory assays (i.e. SRA). False positives are patients in whom immunoassay results were positive, with or without high clinical probabilities that were negative by SRA. Table 2 shows that both the poly-ELISA and IgG-ELISA demonstrated equally high sensitivities (approaching 100\%). The specificity, on the other hand, is lower for the poly-ELISA ( $90.8 \%$ vs. $96.2 \%)$. The IgG-ELISA reclassifies 22 of 29 "borderline" poly-ELISAs into the "negative" category, by IgG-ELISA, resulting in a 75\% reduction in the number of false positives. Consequently, the positive predictive value (PPV) of the IgG-ELISA is higher compared to that of the poly-ELISA (32.0\% vs. $16.3 \%$ ). Both ELISAs were able to detect anti-PF4/heparin antibodies in sera of all true HIT patients.

Table 2 Sensitivity, specificity \& predictive capabilities of immunoassays.

\begin{tabular}{llllll}
\hline \multicolumn{3}{l}{ Using poly } & \multicolumn{5}{l}{ Using IgG } \\
\hline & + & - & & + & - \\
+ & 8 & $4 \mathrm{I}$ & + & 8 & 17 \\
- & 0 & 404 & - & 0 & 428 \\
Sens. & $100.00 \%$ & & Sens. & $100.00 \%$ & \\
Spec. & $90.80 \%$ & & Spec. & $96.20 \%$ & \\
PPV & $16.30 \%$ & & PPV & $32.00 \%$ & \\
NPV & $100.00 \%$ & & NPV & $100.00 \%$ & \\
\hline
\end{tabular}

\section{Discussion}

Our study shows that the IgG-ELISA detected all true HIT patients identified by the poly-ELISA, indicating that utilization of the IgGELISA would not compromise the sensitivity of identifying true HIT. In addition, the IgG-ELISA is able to reclassify a significant number of cases of "borderline positive", by the poly-ELISA, to "negative" by the IgG-ELISA. Thus, while the sensitivity for both immunoassays approaches $100 \%$, the IgG-ELISA demonstrated a higher positive predicative value and specificity than the poly-ELISA. Therefore, labs utilizing the IgG-ELISA may potentially be able to reduce their costs associated with performing the confirmatory testing, and hospitals may also reduce the number of patients receiving unnecessary, expensive and potentially life-threatening direct-thrombin inhibitors prompted by a false positive screening assay for HIT. We also demonstrate that the likelihood of a positive SRA result increases with the OD value for both the poly-ELISA and IgG-ELISA.

HIT is an elusive disorder that once went under-recognized. It is a thrombocytopenic, thromboembolic syndrome instigated by plateletactivating antibodies against PF4/heparin complexes via FcyIIa receptors. ${ }^{9}$ It remains a clinicopathological diagnosis that is initially based on clinical criteria, and later established via the demonstration of PF4/heparin antibodies using laboratory methods (either functional assays or immunoassays). Confirmatory assays are probably the most specific test for HIT with good sensitivity; however, they are technically challenging, some require radioactive reagents, and are thus performed at only a few laboratories resulting in prolonged turnaround times and costly send out tests. ${ }^{10}$ On the other hand, immunoassay kits are available commercially for any lab to perform, are easy and are available at a relatively low cost.

The immunoassays detect antibody binding to complexes of PF4 bound to heparin or heparin-like molecules that have been immobilized to micro titer plates or wells. The advantages of these antigen tests include their availability in kit form, the ease of performance by most hospital laboratories, and the fact that they can provide results in as few as 4 hours from time of specimen acquisition. 
These immunoassays are highly sensitive and therefore have the capacity to detect both clinically insignificant antibodies in addition to the thrombosis-predisposing antibodies. Thus, the specificity of the immunoassays is not as good as the more superior confirmatory laboratory assays (SRA). Since platelets only have Fc $\gamma$ II a receptors (and not IgA or IgM Fc receptors), only PF4/heparin IgG antibodies can bind and activate the platelet. This explains why anti-PF4/heparin $\operatorname{IgM}$ and $\operatorname{IgA}$ antibodies have only a minor relevance in HIT as they have no Fc-part and cannot crosslink the FcyIIa receptor. Therefore, it may be ventured that IgG-ELISAs will perform better than the polyELISA's in assessing for true HIT. There may be rare instances on a case-report level of non-IgG mediated HIT, which may go undetected by the IgG-ELISA. These rare cases are believed to have a different pathogenesis entirely. ${ }^{11,12}$

By analysis of a large, prospective cohort of 453 consecutive patient specimens, we observed that both the poly-ELISA and IgG-ELISA identified PF4/heparin antibodies in all true HIT patients $(n=8)$. Thus, the sensitivity for both types of immunoassays approaches $100 \%$. However, major limitations of these sensitive immunoassays are the risks of over diagnosing the syndrome, particularly in patients who do not have clinical findings compatible with HIT, or in ICU patients where clinical probability can be high but it is due to other causes. Since HIT is known to be dependent on antibody-induced platelet activation, it is a well-recognized limitation of antigen-based immunoassays that although they are highly sensitive, they are less specific in that they are unable to predict activating properties. ${ }^{13}$ In order to confirm activating properties, the functional laboratory assays (or activation tests) are necessary. SRA, the most commonly employed functional release assay in USA (with an estimated sensitivity and specificity as high as $90 \%$ and $100 \%$, respectively, ${ }^{14}$ utilizes washed platelets and detects their activation as a result of antibody binding by measuring aggregation of platelets or release of radio labeled serotonin..$^{15}$ It is chiefly limited by its complexity and the requirement for radioactive reagents. ${ }^{16}$ Consequently, these assays are performed at only a few reference laboratories in the United States. The protracted turn-around time (TAT), additional costs associated with send out testing, and delay in relaying actionable information to clinicians hampers the utility of functional laboratory assays. Therefore, clinicians caring for patients suspected of harboring HIT must rely on the alternate and easier to perform immunoassays to guide their management decisions.

There are two key proposals aimed at improving the positive predictive value of antigen-binding immunoassays:

i. measurement of IgG antibody fraction only, and

ii. consideration of OD values. ${ }^{17}$

The first suggestion is based on evidence that antibodies of the IgM or IgA class have only a minor role in HIT. ${ }^{16,17}$ Direct comparison of poly-ELISA with IgG-ELISA supports this assumption; by the same NPV $(\sim 100 \%)$, the PPV of the IgG-ELISA is significantly higher than poly-ELISA ( $32 \%$ vs. $16 \%$ ) since it reclassifies a larger number of false positives into true negatives. Laboratories performing IgG-ELISAs should report the numerical OD values. Many U.S. laboratories use the same kits (according to CAP surveys), we recommend that laboratories should report the numerical values with the following interpretive ranges for the IgG-ELISAs correlating with the degree of suspicion for true HIT: Negative: $\mathrm{OD}<0.4$, Intermediate positive: $0.4 \leq \mathrm{OD}<1.0$, Positive: $\mathrm{OD} \geq 1.0$. Warkentin et al. ${ }^{2}$ previously recommended a positive $\mathrm{OD}$ value of $\geq 1.0$, which is the value we also recommend, which is slightly lower, and hence more sensitive, than the mean plus one standard deviation derived from our study (OD of 1.2).

The second suggestion comes from laboratory observation: the likelihood of a positive confirmatory test (i.e. SRA) increases with the OD value. There have been some in vitro studies, which have shown that platelet-activation is dependent on the relative quantity of PF4/ hep antibodies. ${ }^{18-22}$ Warkentin et al. ${ }^{2}$ have shown that the probability of a strongly positive SRA is less than or equal to $5 \%$ in cases with an IgG PF4 OD value of 0.40-1.00. They also demonstrated that for every 1.0 U increase in OD, the probability of a positive SRA increases by 40 (odds ratio), or, expressed another way, from $<5 \%$ to $\sim 25 \%$ to $\sim 95 \%$ for positive OD values of $0.5,1.5$, and 2.5 , respectively. ${ }^{23}$, ${ }^{24}$ Using a comparison of patients with poly-ELISA results, IgGELISA results, SRA results, and clinical suspicion of HIT, this range was verified in our study. Twelve (12) patient samples with clinical suspicion of HIT and IgG-ELISA values in the borderline range (OD 0.4-1.0) were tested via SRA and all 12 were negative by SRA $(100 \%)$. Fourteen (14) patient samples with clinical suspicion of HIT and positive IgG-ELISA values (OD $>1.0)$ were tested for SRA and eight (8) of those samples were positive for SRA (57.1\%). Therefore, while the IgG-ELISA can improve positive predictive value, it still remains a screening assay. In order to prevent the over diagnosis of HIT, a confirmatory functional assay (such as SRA) is still necessary.

\section{Conclusion}

A practical diagnostic approach to HIT will have to include several aspects. First and foremost, a so-called "pre-test" scoring system of clinical signs and symptoms should be applied to all patients suspected of harboring HIT. There are currently a few different models in existence; however, we prefer the Warkentin et al. ${ }^{2}$ 4T's scoring system. These "pre-test" scoring systems can filter patients into low, intermediate, and high pretest categories. We believe that patients with a low pretest probability should NOT be evaluated with laboratory tests, as some assays (poly-ELISA) will demonstrate a positive result not withstanding the absence of HIT.

Second, an IgG-ELISA is of greater diagnostic utility than a poly-ELISA. Sera from patients with an intermediate to high pretest probability of HIT should be tested with an IgG-ELISA, which results in less false positive, or "borderline positives" when compared to the poly-ELISA. Third, in case of a positive result, confirmatory testing should be performed, especially at institutions that care for severely ill patients where false positive rates can be higher, in our hospital, the above proposals were successfully implemented in 2006 with the exception of the utilization of a poly-ELISA. As a result of the current study, we now employ the IgG-ELISA overseen by a team of expert clinical pathologists, pathology residents, and pharmacists.

\section{Acknowledgements}

None.

\section{Conflict of interest}

The author declares no conflict of interest.

\section{References}

1. Girolami B, Prandoni P, Stefani PM, et al. The incidence of heparininduced thrombocytopenia in hospitalized medical patients treated with subcutaneous unfractionated heparin: A prospective cohort study. Blood. 2003;101(8):2955-2959. 
2. Warkentin TE, Davidson BL, Buller HR, et al. Prevalence and risk of pre-existing heparin-induced thrombocytopenia antibodies in patients with acute VTE. Chest. 2011;140(2):366-373.

3. Levine RL, Hergenroeder GW, Francis JL, et al. Heparin-platelet factor 4 antibodies in intensive care patients: an observational seroprevalence study. J Thromb Thrombolysis. 2010;30(2):142-148.

4. Berry C, Tcherniantchouk O, Ley EJ, et al. Overdiagnosis of heparininduced thrombocytopenia in surgical ICU patients. J Am Coll Surg. 2011;213(1):10-17.

5. GreinacherA, Juhl D, Strobel U, et al. Heparin-induced thrombocytopenia: a prospective study on the incidence, platelet-activating capacity and clinical significance of anti-platelet factor4/heparin antibodies of the IgG, IgM, and IgA classes. J Thromb Haemost. 2007;5(8):1666-1673.

6. Juhl D, Eichler P, Lubenow N, et al. Incidence and clinical significance of anti-PF4/heparin antibodies of the $\operatorname{IgG}, \operatorname{IgM}$, and $\operatorname{IgA}$ class in 755 consecutive patient samples referred for diagnostic testing for heparininduced thrombocytopenia. Eur J Haematol. 2006;76(5):420-426.

7. Lindhoff-Last E, Gerdsen F, Ackermann H, et al. Determination of heparin-platelet factor 4-IgG antibodies improves diagnosis of heparininduced thrombocytopenia. Br J Haematol. 2001;113(4):886-890.

8. Berry C, Tcherniantchouk O, Ley EJ, et al. Overdiagnosis of heparininduced thrombocytopenia in surgical ICU patients. J Am Coll Surg. 2011;213(1):10-17.

9. Greinacher A. Heparin-induced thrombocytopenia: frequency and pathogenesis. Pathophysiol Haemost Thromb. 2006;35(1-2):37-45.

10. McFarland J, Lochowicz A, Aster R, et al. Improving the specificity of the PF4 ELISA in diagnosing heparin-induced thrombocytopenia. Am J Hematol. 2012;87(8):776-781.

11. Ziporen L, Li ZQ, Park KS, et al. Defining an antigenic epitope on platelet factor 4 associated with heparin-induced thrombocytopenia. Blood. 1998;92(9):3250-3259.

12. Amiral J, Meyer D. Heparin dependent antigens in heparin induced thrombocytopenia. In: Warkentin TE, et al. editors. Heparin-Induced Thrombocytopenia and Thrombosis. New York: Marcel-Dekker; 2004. p. $165-178$.

13. Amiral J, Pouplard C, Vissac AM, et al. Affinity purification of heparindependent antibodies to platelet factor 4 developed in heparin-induced thrombocytopenia: Biological characteristics and effects on platelet activation. Br J Haematol. 2000;109(2):336-341.
14. Kelton JG, Smith JW, Warkentin TE, et al. Immunoglobulin G from patients with heparin-induced thrombocytopenia binds to a complex of heparin and platelet factor 4. Blood. 1994;83(11):3232-3239.

15. Visentin GP, Ford SE, Scott JP, et al. Antibodies from patients with heparin-induced thrombocytopenia/thrombosis are specific for platelet factor 4 complexed with heparin or bound to endothelial cells. $J$ Clin Invest. 1994;93(1):81-88.

16. Greinacher A, Althaus K, Krauel K, et al. Heparin-induced thrombocytopenia. Hamostaseologie. 2010;30(1):17-18, 20-28.

17. Amiral J, Wolf M, Fischer A, et al. Pathogenicity of IgA and/or IgM antibodies to heparin-PF4 complexes in patients with heparin-induced thrombocytopenia. Br J Haematol. 1996;92(4):954-959.

18. Meyer O, Aslan T, Koster A, et al. Report of a patient with heparininduced thrombocytopenia type II associated with IgA antibodies only. Clin Appl Thromb Hemost. 2006;12(3):373-375.

19. Warkentin TE, Sheppard JA, Moore JC, et al. Laboratory testing for the antibodies that cause heparin-induced thrombocytopenia: how much class do we need? J Lab Clin Med. 2005;146(6):341-346.

20. Arepally G, Reynolds C, Tomaski A, et al. Comparison of PF4/heparin ELISA assay with the $14 \mathrm{C}$-serotonin release assay in the diagnosis of heparin-induced thrombocytopenia. Am J Clin Pathol. 1995;104(6):648654 .

21. Warkentin TE, Sheppard JA. Testing for heparin-induced thrombocytopenia antibodies. Transfus Med. 2006;20(4):259-272.

22. McFarland J, Lochowicz A, Aster R, et al. Improving the specificity of the PF4 ELISA in diagnosing heparin-induced thrombocytopenia. Am J Hematol. 2012;87(8):776-781.

23. Zwicker J, Uhl L, Huang WY, et al. Thrombosis and ELISA optical density values in hospitalized patients with heparin-induced thrombocytopenia. $J$ Thromb Haemost. 2004;2(12):2133-2137.

24. Khairy M, Lasne D, Brohard-Bohn B, et al. A new approach in the study of the molecular and cellular events implicated in heparin-induced thrombocytopenia. Formation of leukocyte-platelet aggregates. Thromb Haemost. 2001;85(6):1090-1096. 